\title{
REVEALING LOCAL VARIABILITY PROPERTIES OF HUMAN HEARTBEAT INTERVALS WITH THE LOCAL EFFECTIVE HÖLDER EXPONENT
}

\author{
ZBIGNIEW R. STRUZIK \\ Centre for Mathematics and Computer Science (CWI), \\ P.O. Box 94079, $1090 \mathrm{~GB}$ Amsterdam, The Netherlands \\ E-mail: Zbigniew.Struzik@cwi.nl
}

Received July 17, 2000; Accepted September 4, 2000

\begin{abstract}
The local effective Hölder exponent has been applied to evaluate the variability of heart rate locally at an arbitrary position (time) and resolution (scale). The local effective Hölder exponent ${ }^{1,2}$ used is effectively insensitive to local polynomial trends in heartbeat rate due to the use of the Wavelet Transform Modulus Maxima (WTMM) technique. Also the variability so obtained is compatible in the sense of distribution to the multifractal spectra of the analyzed heart rate time series. This provides the possibility of standardizing the variability estimation for comparison between different patients and between different recordings for one patient. The previously reported global correlation behavior ${ }^{3}$ is captured in the effective Hölder exponentbased, local variability estimate. This includes discriminating healthy and sick (congestive heart failure patients) on the basis of both the central (Hurst) exponent and the width of the multifractal spectra. In addition to this, we observed intriguing patterns of individual response in variability records to daily activities. A moving average filtering of Hölder exponent-based variability estimates was used to enhance these fluctuations. We find that this way of local presentation of scaling properties may be of clinical importance.
\end{abstract}

\section{INTRODUCTION}

Recent findings reported in Ivanov et al. (1999) ${ }^{3}$ suggest that heartbeat rate is more complex than has been anticipated, requiring multiple, densely interwoven scaling (roughness) exponents to describe it. This is in contrast to the more established view which associated one global roughness 
exponent with each heartbeat record. ${ }^{4}$ This exponent, of course, could be different for various people and also depended on the state of health. ${ }^{5-8}$ A multifractal description reveals exponents changing from point to point in a way which suggests some higher order organization. Such multifractal exponents cannot be simply grouped in patches of constant or stationary behavior, but display complex, nonstationary structure at any time scale (resolution) considered.

The multifractality discovery was made possible by the application of the wavelet transform modulus maxima (WTMM) method in the multifractal analysis of the heartbeat signal. The formalism, developed by Arneodo et al. in the early nineties has been successfully used to test many natural phenomena. ${ }^{9-15}$ One of the key aspects of this methodology is that it is intrinsically statistical and provides only global estimates of scaling (of the moments of relevant quantity). While this is often a required property, in some cases local information about scaling may be more relevant than the global spectrum. This is particularly true for time series where scaling properties are nonstationary, whether it be due to intrinsic changes in the signal scaling characteristics, noise or simply the boundary effects.

To address this problem, we have introduced ${ }^{1,2}$ a method of estimation of the local scaling exponent through the paradigm of the multiplicative cascade. We reveal the hierarchy of the scaling branches of the cascade with the WTMM tree, which has proved to be an excellent tool for the purpose. ${ }^{10,22}$ Contrary to the intrinsically unstable local slope of the maxima lines, this estimate is robust and provides a stable, effective Hölder exponent, local in scale and position.

For model multifractals, the mechanism behind multiscale nonstationarity in the roughness exponent values does not change with resolution (scale). It can be captured in a distribution (or spectrum) of multifractal exponents, which for idealized multifractal signals shows the same spectrum independent of the length or starting position of the investigated time series. Such behavior is typical for model multifractals, but in heartbeat and in other real life signals, changes are observed in all spectral characteristics: positioning, shape and modality, depending on the time series investigated, but also on the selected time and scale range of analysis.
While this adds to the complexity of the global (spectral) description, it also validates the local approach, where we choose to look back at the temporal organization of the scaling (effective Hölder) exponent in the hope that the analysis of its intrinsic nonstationarities will provide insight into global behavior. ${ }^{\text {a }}$

In this paper, we applied the local effective Hölder exponent to evaluate the variability of the heartbeat rate locally at an arbitrary position (time) and resolution (scale). Just as is the case in global WTMM based multifractal formalism, the technique is effectively insensitive to local polynomial trends in heartbeat rate. Also the variability so obtained is compatible in the sense of distribution to the multifractal spectra of the analyzed heartbeat rate time series.

Therefore, the previously reported global correlation behavior is captured in the effective Hölder exponent based, local variability estimate. This includes discriminating healthy and sick (congestive heart failure patients) on the basis of both the central (Hurst) exponent $t^{5}$ and the width of the multifractal spectra. ${ }^{3}$ However, in addition to this, we observed intriguing patterns of individual response (nonstationarities) in variability records to daily activities and during sleep.

The observation of nonstationarity of the variability estimate obtained with the effective Hölder exponent prompted us to conduct a series of experiments. The main objective of the tests was to develop a methodology capable of answering the following questions:

(1) Is the observed multifractal behavior of the heartbeat the result of nonstationarity of the local effective Hölder exponent?

(2) Can the nonstationarity be linked with activity, i.e. the particular mental or somatic state of the person?

(3) In the case of known physiological input like $\beta$ blocker, is the nonstationarity affected?

(4) In the case of non-activity such as during sleep, is the nonstationarity still observed?

Of course all the above questions lead to the central question of the physiological reasons for the apparent multifractal behavior of the heartbeat. We will not attempt to provide a detailed answer in this mainly methodological paper. Rather, we will show

\footnotetext{
a For example, into the way the spectrum is built.
} 
that the methods described can give answers to particular questions of the kind listed above. We therefore hope that the methodology presented can help, through extensive study, in understanding systems characterized by apparently multifractal behavior.

The structure of the paper is as follows. In Sec. 2, we focus on the relevant aspects of the wavelet transformation, in particular the ability to characterize scale-free behavior through the Hölder exponent. Together with the hierarchical, scalewise decomposition provided by the wavelet transform, it will enable us to reveal the scaling properties of the tree of the multiplicative cascading process. In Sec. 3, we briefly describe a technical model enabling us to estimate the scale-free characteristic (the effective Hölder exponent) for the branches of such a process. A more extensive coverage of this method is available in Refs. 1 and 2. In Sec. 4, we use the derived effective Hölder exponent for the local temporal description of the various test heartbeat time series. Section 5 provides an extension to fluctuation analysis of the effective Hölder exponent. Again a number of test signals are used to illustrate the effectiveness of the method and address the central questions arising from the nonstationarity of local effective Hölder exponent. Section 6 closes the paper with conclusions.

\section{CONTINUOUS WAVELET TRANSFORM AND ITS MAXIMA USED TO REVEAL THE STRUCTURE OF SINGULARITIES IN THE TIME SERIES}

Conceptually, the wavelet transformation ${ }^{16-18}$ is a convolution product of the time series with the scaled and translated kernel - the wavelet $\psi(x)$, usually a $n$th derivative of a smoothing kernel $\theta(x)$. Usually, in the absence of other criteria, the preferred choice is the kernel, since it is well localized both in frequency and position. In this paper, we chose the Gaussian $\theta(x)=\exp \left(-x^{2} / 2\right)$ as the smoothing kernel, since it provides wavelets with optimal localization in both domains.

The scaling and translation actions are performed by two parameters; the scale parameter $s$ "adapts" the width of the wavelet kernel to the res- olution required and the location of the analyzing wavelet is determined by the parameter $b$ :

$$
W f(s, b)=\frac{1}{s} \int_{-\infty}^{\infty} d x f(x) \psi\left(\frac{x-b}{s}\right)
$$

where $s, b \in \mathbf{R}$ and $s>0$ for the continuous version (CWT).

The 3D plot in Fig. 1 shows how the wavelet transform (WT) reveals more and more detail while going towards smaller scales, i.e. towards smaller $\log (s)$ values. The WT is sometimes referred to as the "mathematical microscope", 9 due to its ability to focus on weak transients and singularities in the time series. The wavelet used determines the optics of the microscope; its magnification varies with the scale factor $s$.

This property makes the continuous wavelet transform very useful in analyzing local regularity (scaling/roughness) properties of functions. In particular, such local scaling behavior is often characterized by the Hölder exponent $h$. The following scaling equation defines the Hölder exponent $h\left(x_{0}\right) \in(n, n+1)$ of the cusp singularity at $x_{0}$ :

$$
\left|f(x)-P_{n}\left(x-x_{0}\right)\right| \leq C\left|x-x_{0}\right|^{h}
$$

as the supremum of all $h$ such that the above relation holds for some polynomial $P_{n}$ of degree $n<h$. $P_{n}$ can often be associated with the Taylor expansion of $f$ around $x_{0}$, but Eq. (2.1) is valid even if such an expansion does not exist. ${ }^{19}$ The Hölder exponent is, therefore, a function defined for each point of $f$, and it describes the local regularity of the function (or distribution) $f$.

It can be shown ${ }^{20}$ that for cusp singularities, the location of the singularity can be detected, and the related exponent can be recovered from the scaling of the WT, along the so-called maxima line, converging towards the singularity. This is a line where the wavelet transform reaches local maximum (with respect to the position coordinate). Connecting such local maxima within the continuous WT "landscape" gives rise to the entire tree of maxima lines. Restricting oneself to the collection of such maxima lines provides a particularly useful representation ${ }^{21}$ of the entire CWT, the so-called WTMM representation. It incorporates the main characteristics of the WT: the ability to reveal the hierarchy of (singular) features, including the scaling behavior. In particular, we have the following power law proportionality for the wavelet transform of the cusp singularity in $f\left(x_{0}\right)$ :

$$
W^{(n)} f\left(s, x_{0}\right) \sim|s|^{h\left(x_{0}\right)} .
$$




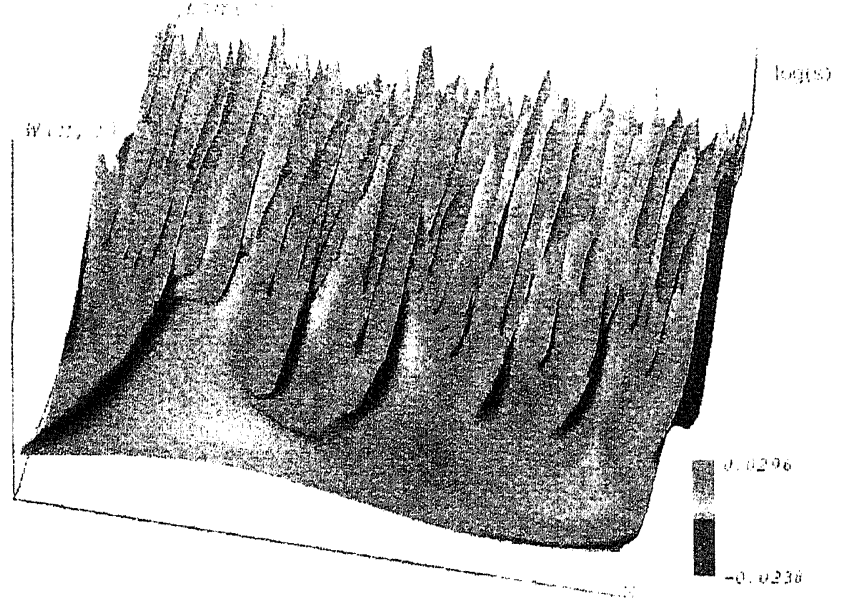

(a)

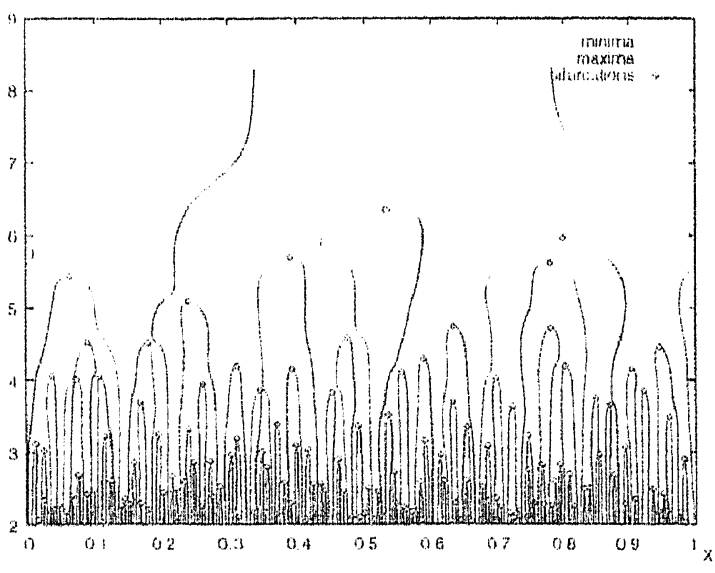

(b)

Fig. 1 (a) Representation of the random walk (Brownian process) time series. The wavelet used is the Mexican hat - the second derivative of the Gaussian kernel. The coordinate axes are: position $x$, scale in logarithm $\log (s)$, and the value of the transform $W(s, x)$. (b) The related WTMM representation.

This is under the condition that the wavelet has at least $n$ vanishing moments, i.e. it is orthogonal to polynomials up to degree $n: \int_{-\infty}^{+\infty} x^{m} \psi(x) d x=0$ $\forall m, 0 \leq m<n$. The reader will note that this requirement is needed to filter the polynomial $P_{n}$ in Eq. (2.1) in order to access the unbiased scaling exponent.

An additional benefit of using the WT is that we are not limited to the continuous function $f$ in the analysis of local regularity, Eq. (2.2). Through the convolution with continuous kernels, we can also analyze distributions or, in particular, discretely sampled inputs. As a consequence, the exponent $h$ can take negative values. For exarmple, as can be quickly verified from $\mathrm{Eq}_{\mathrm{q}}$. (2.0), for the Dirac pulse $D\left(x_{0}\right)$, we have $W\left(D\left(x_{0}\right)\right)=1 / \mathrm{s}$. Thus $h_{\text {Dirac }}=-1$.

\section{ESTIMATION OF THE LOCAL, EFFECTIVE HÖLDER EXPONENT USING THE MULTIPLICATIVE CASCADE MODEL}

We have shown in the previous section that the WT, and in particular its maxima lines, can be used in evaluating the Hölder exponent in isolated singularities. In most real-life situations, however, the singularities in the time series are not isolated but densely packed. The logarithmic: rate of increase or decay of the corresponding WT maximum line is usually not stable but fluctuates, following the action of some process involved, see Fig. 2(a) for a typical example.

To capture the fuctuations and estimate the related exponents (to which we will refer to as an effective Hölder exponent of the singularity), we will model the singularities as created in some kind of a collective process of a very generic class -.... the multiplicative cascade model. Each point of this cascade is uniquely characterized by the sequence of indices $\left(s_{1} \cdots s_{n}\right)$, taking index values from the set of weights $\left\{p_{i}\right\}$. The sequence indicates the unique order in which the woights are successively acting along the process branch leading to the particular singularity.

Suppose that we denote the density of the cascade at the generation level $F_{i}$ ( $i$ running from 0 to $\max )$ by $r\left(F_{i}\right)$, we then have

$$
\kappa\left(F_{\text {max }}\right)=p_{s_{1}} \cdots p_{s_{n}} k\left(F_{0}\right)=P_{F_{0}}^{F_{\text {max }}} \kappa\left(F_{0}^{\prime}\right)
$$

and the local exponent is related to the rate of increase of the product $P_{F_{\text {max }}}^{F_{\text {max }}}$ over the gained scale difference. In any experimental situation, the weights $p_{i}$ are not known and $h$ has to be estimated. This can be simply clone using the fact that for the multiplicative cascacle process, the effective product 


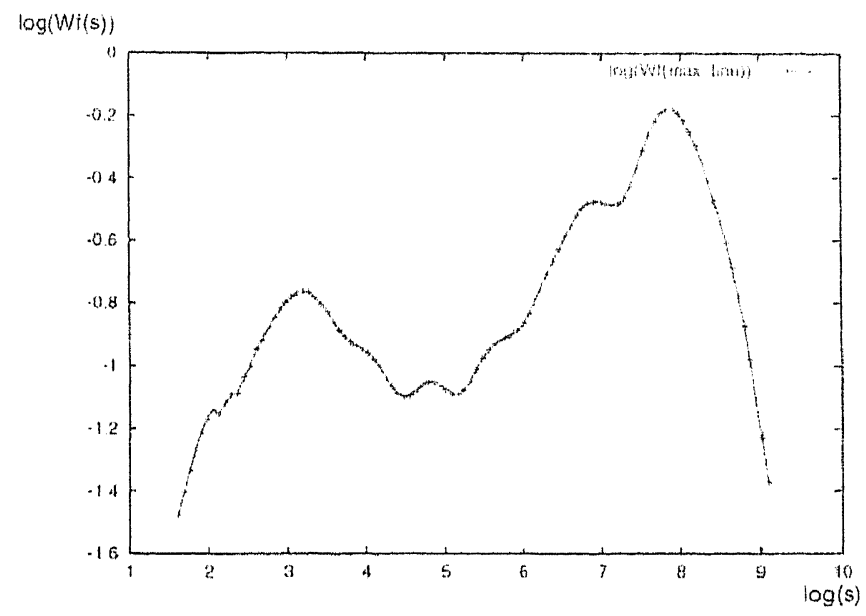

(a)

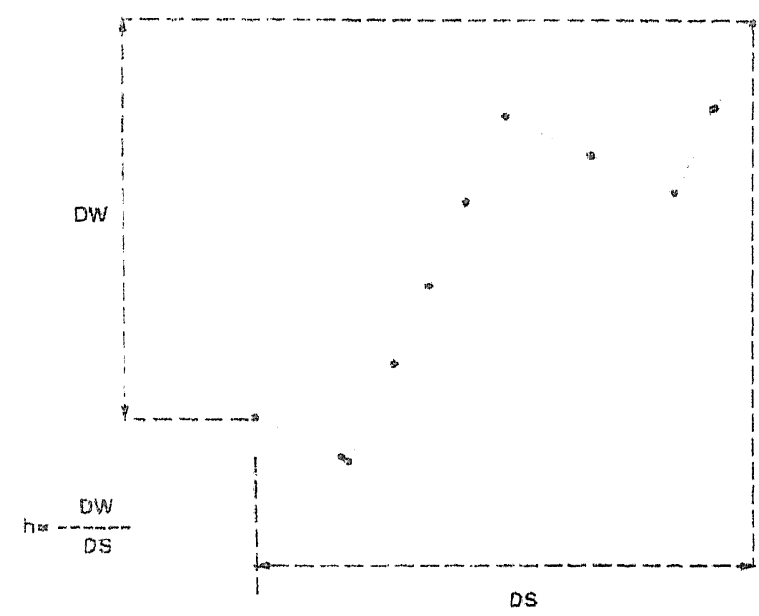

(b)

Fig. 2 (a) It is impossible to evaluate the scaling exponent for an arbitrary maximum line participating in a complex process: a real life example of a maximm line. (b) The local effective Hölder exponent estimate takes the effective difference in the logarithm of the density of the process with respect to the logarithm of the scale difference gained along the process path.

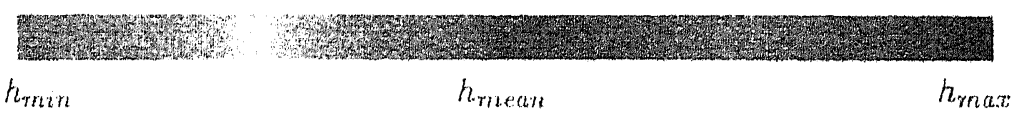

Fig. 3 Color range for the strips of the effective Hölder exponent.

of the weighting factors is reflected in the difference of logarithmic values of the densities at $F_{0}$ and $F_{\max }^{\prime}$ along the process branch:

$$
h_{F_{\max }}^{F_{0}}=\frac{\log \left(\kappa\left(F_{\max }\right)\right)-\log \left(\kappa\left(F_{0}\right)\right)}{\log \left((1 / 2)^{\max }\right)-\log \left((1 / 2)^{0}\right)} .
$$

The densities along the process branch can be estimated with the WT, using its remarkable ability to reveal the entire process tree of a multiplicative process. ${ }^{22,23}$ It can be shown that the densities $k\left(F_{i}\right)$ correspond with the value of the WT along the maxima lines belonging to the given process branch. The estimate of the effective Hölder exponent becomes:

$$
\hat{h}_{s_{l o}}^{s_{h i}}=\frac{\log \left(W \int \omega_{p b}\left(s_{l o}\right)\right)-\log \left(W f \omega_{p b}\left(s_{h i}\right)\right)}{\log \left(s_{l o}\right)-\log \left(s_{h i}\right)}
$$

where $W f \omega_{p b}(s)$ is the value of the WT at the scale $s$, along the maximum line $\omega_{p b}$ corresponding to the given process branch $b p$. Scale $s_{\text {lo }}$ corresponds with generation $F_{\max }$, while $s_{h i}$ corresponds with generation $F_{0}$ (simply the largest available scale in our case), sec Fig. 2(b). ${ }^{\text {b }}$

\section{EMPLOYING THE LOCAL EFFECTIVE HÖLDER EXPONENT IN THE CHARACTERIZATION OF HEARTBEAT INTERVAL TIME SERIES}

Such an estimated local $\hat{h}\left(x_{0}, s\right)$ can be depicted in a temporal fashion, for example with color stripes as we have done in Fig. 3. The color of the stripes is determined by the value of the exponent $\hat{h}\left(x_{0}, s\right)$ and its location is simply the $x_{0}$ location of the analyzed singularity (in practice this amounts to the location of the corresponding maximum line). Color coding is done with respect to the moan value, which is set to the green color central to our rainbow range.

\footnotetext{
${ }^{b}$ For $s_{t o}$ we will use $a=5$ in the examples presented in this paper. This is the lowest resolution for which we can maintain the shape of the Mexican hat.
} 

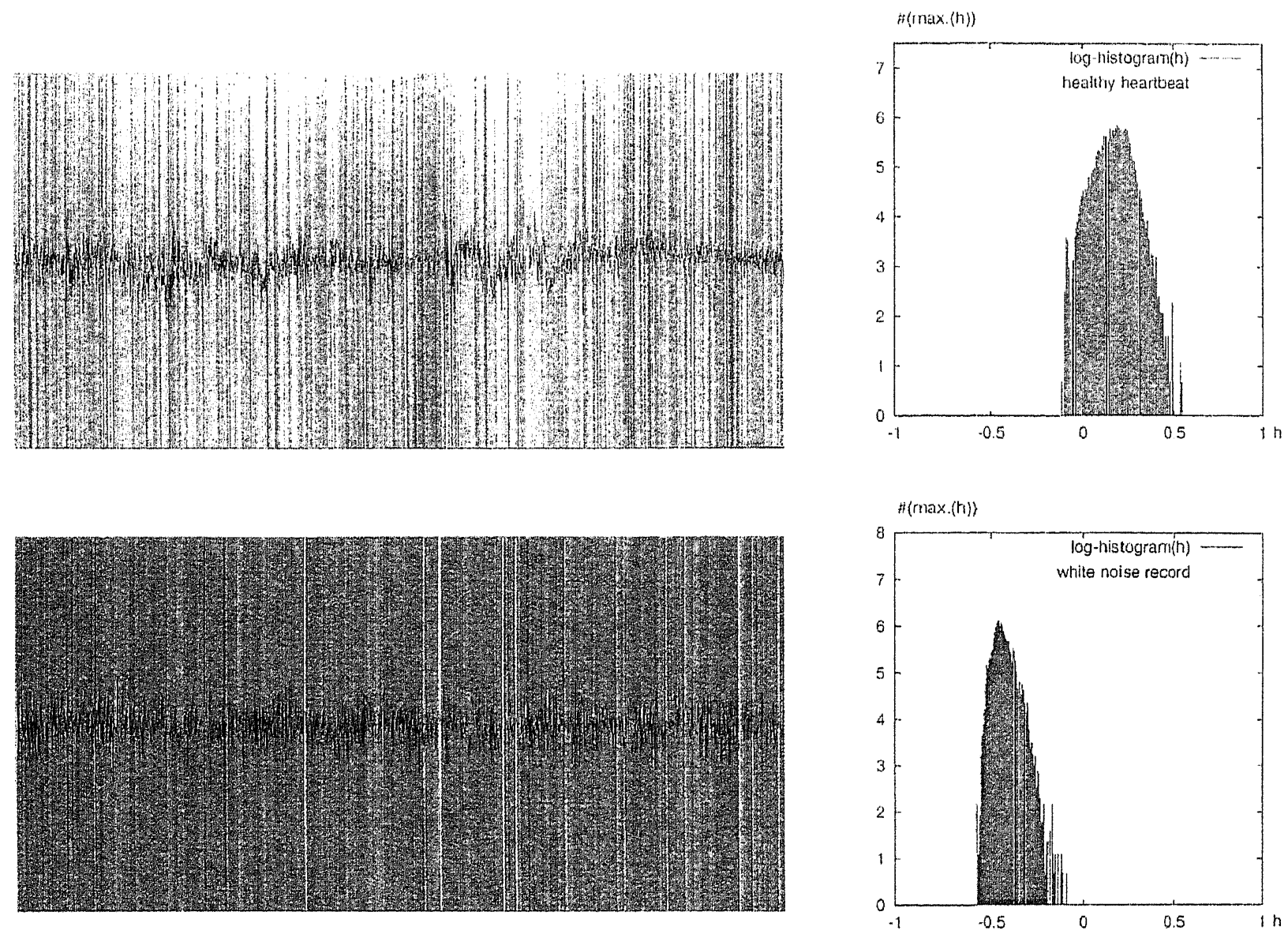

(a)

(b)

Fig. 4 (a) Example time series with local Hurst exponent indicated in color: the record of healthy heartheat intervals (above) and white noise (below). The background color indicates the Hölder exponcnt locally, centered at the Iurst exponent at green; the color goos towards blue for higher $\hat{h}_{h}$ and towards red for lower $\hat{h}$. (b) The corresponding log-histograms of the local Hölder cxponcutt.

All exponent values lower than the mean value are given colors from the "warmer" side of the rainbow, all the way towards dark rel. All higher than average exponents get "colder" colors, down to dark blue.

The first example in Fig. 4(a) is a record 2.4 of heartbeat intervals recorded from a healthy human heart and it shows an intricate structure of interwoven singularities at various strengths. This bohavior has becn recently reported ${ }^{3}$ to correspond with the multifractal behavior of the heartbeat. The green is centered at $\hat{h}_{l}=0.1$ for this pariel. The second example time series is a computer-generated sample of white noise. It shows almost monochromatic behavior, centered at $h_{l}=-0.5$. $^{\mathrm{C}}$ The color green is clominant. There are, however, several instances of darker green and light, blue, indicating locally sinooth components.

In Fig. 4(b), the log-histograms are shown of the Hölder exponent displayed in the color pancls. They

\footnotetext{
The negative exponent for white noise (contral, mean value) $h=-0.5$ is perfectly admissible for a time series in the generalized formulation, and can be measured with the WT, see Eq. (2.2). It is also consistent with the Flurst exponent $H=0.5$ for the random walk - the integral of white noise: $H=h_{\text {random walk }}=h_{\text {white nuise }}+1$.
} 


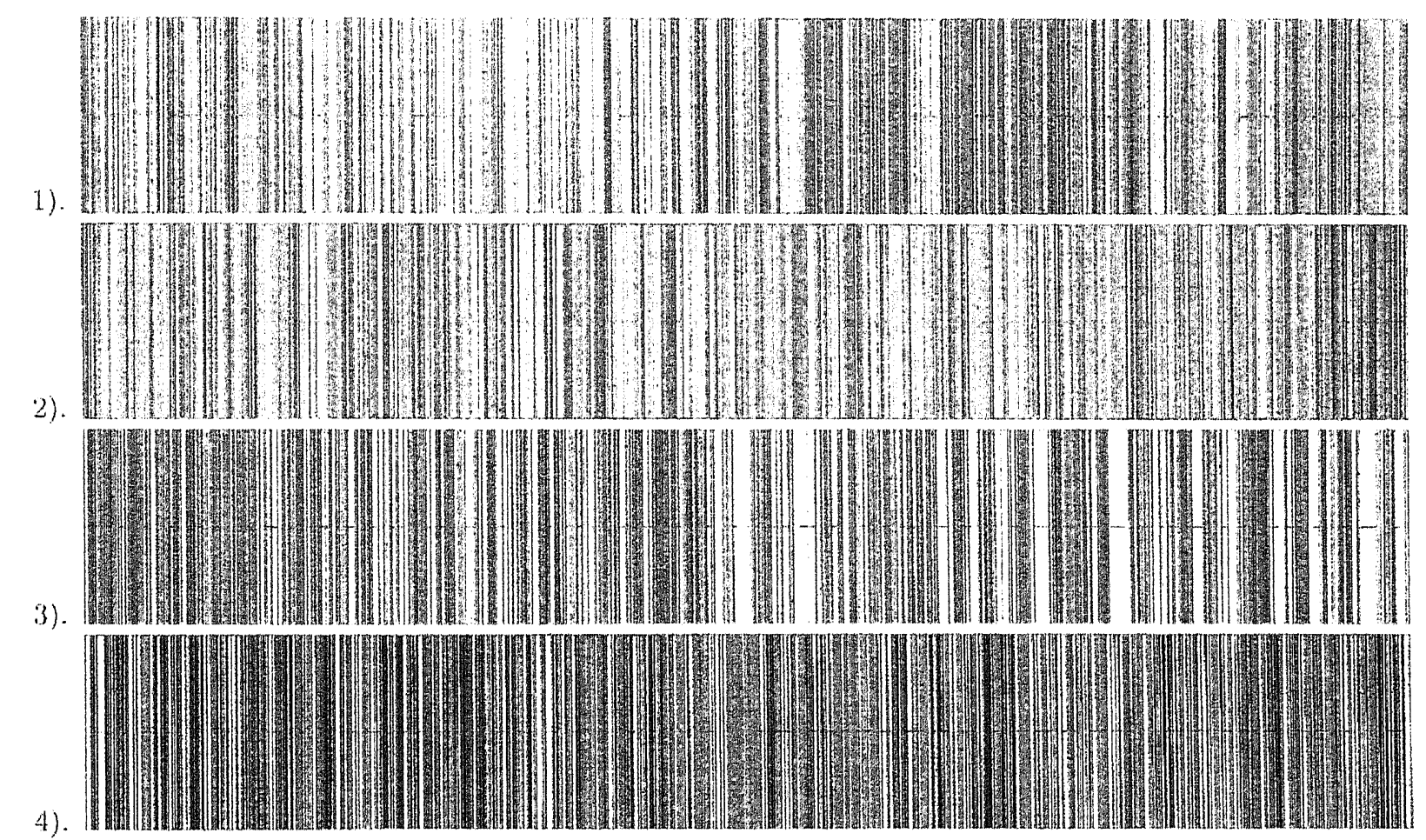

Fig. 5 Local Hurst exponent indicated in color for four hear tbeat interval time series (not shown). The green color is centered at $h=0.0$ for all plots. It saturates at dark blue for $h=0.4$ and at dark red for $h=-0.4$.

are made by taking the logarithm of the measure in each histogram bin. This conserves the monotonicity of the original histogram, but allows us to compare the log-histograms with the spectrum of singularities $D(h)$. The log-histograms are actually closely related to the (multifractal) spectra of the Hölder exponent. ${ }^{2}$ The multifractal spectrum of the Hölder exponent is the "limit histogram" $D_{s \rightarrow 0}(h)$ of the Hölder exponent in the limit of infinite resolution. Of course, we cannot speak of such a limit other than theoretically, and therefore, a limit histogram (multifractal spectrum) has to be estimated from the evolution of the log-histograms along scale. For details, see Struzik (2000). ${ }^{2}$

The ability to display the scaling properties of the tirne series in a local manner has already proven quite successful. We have applied the method to a set of heartbeat interval time series in a (double) blind test. The purpose of the test was to establish whether the local effective Hölder exponent "panels" can be used in discerning whether the time series is from a healthy or an ill subject.
In Fig. 5, we show one such set of panels. There are two records of healthy heartbeats and two of heart disease. The center value of the effective Hölder exponent is $h_{2}=0.1$, and is displayed in green calor. For $h>0.1$, the color becomes darker green, through light blue, it saturates as dark blue for $h=0.5$. All $h>0.1$ correspond with a higher than average local degree of correlation, which has been associated with heart failure. On the contrary, all $h<0.1$ indicate stronger than average (relative) anti-correlation, which can be associated with healthy heartbeat behavior. The colors displayed for $h<0.1$ are from light green through yellow and orange towards red at $h=-0.3$.

The two upper panels (1) and (2) in Fig. 5 both belong to healthy individuals but of course there is no reason why they should be iclentical; and indeed individual patterns all have a different arrangement of color stripes. Also the density of colors changes; some nonstationary behavior is apparent in the Fig. 5, panels (1) and (2). Both panels show a wide range of colors reflecting fully 
developed dyamies of the Lealty hetrtbeat. Such panels correspond to a wide multifractal spectrum:"

The two lower panels (3) and (4), on the contrary, are taken from heartbeats of congestive heart failure (CHF) cases. The deviation from healthy behavior can occur in a number of ways but these two are perhaps the most generic. One lype of deviation causes a narrowing of the dynamics and therefore a narrowing of the range of colors - it is reminiscent of the monofractal noise shown in Fig. 4. The other type of deviation, in addition to a narrower color spectrum, is that it drifted away in its entire? color spectrum in the direction of green and blue, that is towards smoother (more correlated) behavior. This shift in the central color reflects the change in the global correlation exponent between healthy and CHF individuals. 5

We have left the background between the stripes uncolored (white) although it corresponds with even more smooth regions (at the resolution considered!) and can all be painted dark blue. This would enhance the color range and help diagnosis, but we preferred not to introduce any additional information to the panels in this research paper.
It is apparent that the color panels provide more information than the global averages, like the central color or the range of colors used, i.e. the width of the multifractal spectrurn. In the following, we will show how to exploit this additional information pertinent to the local color fluctuations (nonstationarities) in the panels.

\section{COLLECTIVE PROPERTIES OF THE LOCAL VARIABILITY ESTIMATE}

In the context of heartbeat, one may ask the question: what is the meaning of the varying local scaling exponent. Also, its temporal organization and its relevance to the multifractal spectrum may be tested. Ultimately, we would like to identify physiological reasons for the apparent multifractal behavior of the heartbeat. Let us first go back to numerical information, the Hölder exponent which we used to represent in color, and exploit a few generic cases of nonstationarity.

In Fig. 6, three exaruple heartbeat interval time series are shown with their corresponding local
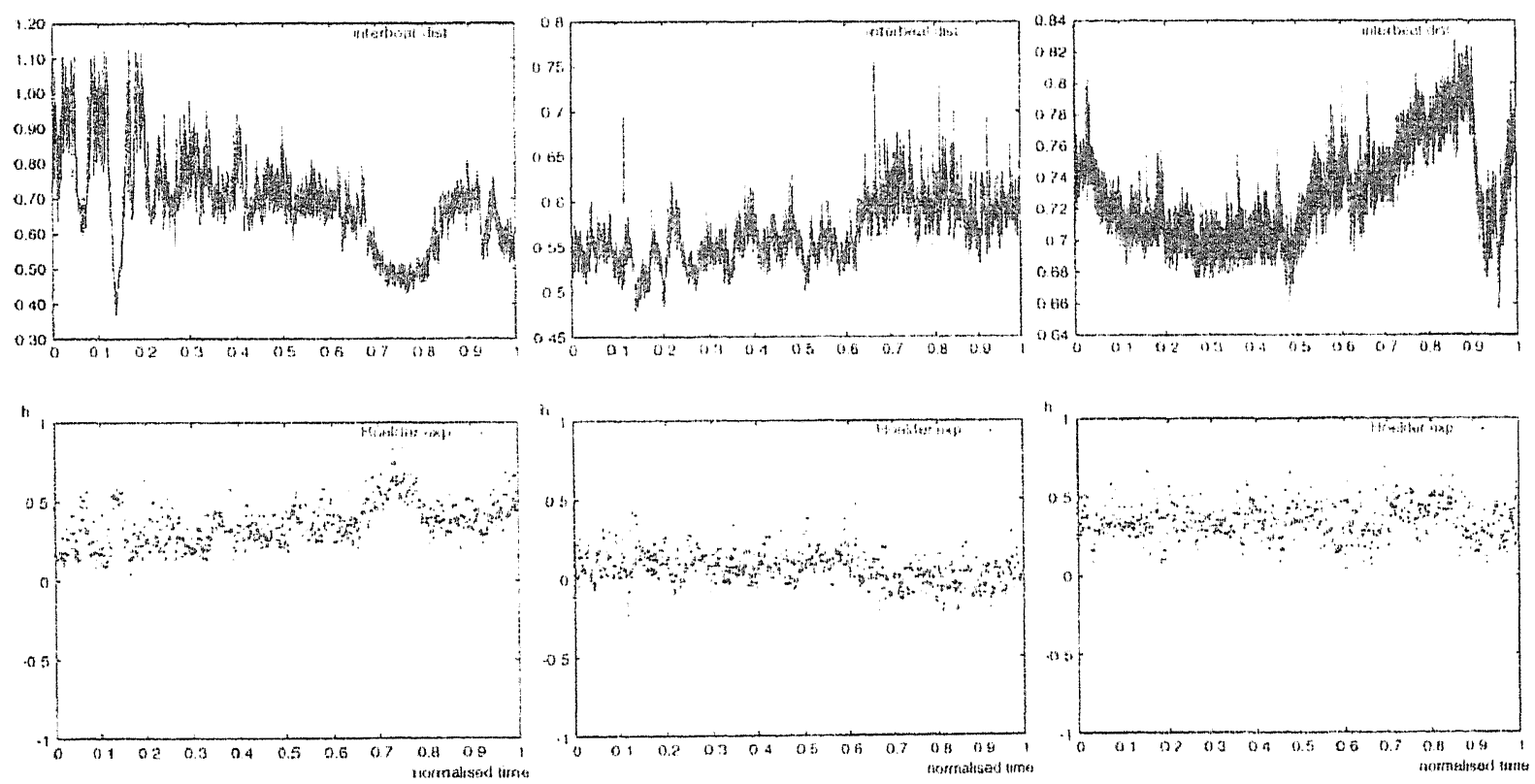

Fig. 6 Three example heartbeat interval tine series (top row) with their corresponding local effective Hölder exponent (bottorn row). Two examples of nonstationarities in local Hölder exponent; they are intrinsic to the local Hölder exponent not to the nonstationarities of the input time series, as is shown in the third example, showing independence of the polynomial trend in the input. 
effective Hölder exponent. The first example from the left shows a consistent linear trend - the increase of the exponent value with time (bottorn plot). This can also be verified in the corresponding time series above (leftmost, top plot): the roughness of the time series decreases with time (except for some minor fluctuations). In the second example (center panel), two distinct regimes can be distinguished with a somewhat different mean $h$ value: one region up until sample 5000 and the other of slightly lower value of $h$ for samples 5500 and more. Both the above cases will result in a broadoning of the $D(h)$ spectrum as a result of the nonstationary behavior of $h$. This effect alone, if observed at one global resolution, would not be sufficient for multifractal behavior - it would simply mean that the local variability is nonstationary in these time series. In the following, we will detect multiscale nonstationarity of $h$. In the last cxample in the rightmost panels, we show that the $h$ exponent and the roughness of the time series are independent of the fluctuations or trends in the time series.

The evident nonstationary behavior in these figures carn be quartified, and for this purpose we used a low pass rnoving average filter (MA) to detect/enhance trends. This processing is, of course, done on the Hölder exponent value set $\left\{h_{i}(f(x))\right\}$, not on the input signal $f(x)$. A $n$-MA filtering of $n$ base is defined as follows:

$$
h_{\mathrm{MA}_{n}}(i)=\frac{1}{n} \sum_{i=1}^{i=n} h_{i}(f(x))
$$

where $h_{i}(f)$ are the subsequent values of the effective Hölder exponent of the time series $f$. Standard deviation from the $h_{\mathrm{MA}_{r}}$ (i) mean exponent can also be calculated:

$$
S D h_{\mathrm{MA}_{n}}(i)=\frac{1}{n} \sqrt{\sum_{i=1}^{i=\tau_{2}}\left(h_{n_{i}}(f(x))-h_{\mathrm{MA}_{n}}(i)\right)^{2}} .
$$

The observation of nonstationarity of the variability estimate obtained with the effective Hölder exponent prompted us to do a series of experiments. The main objective of the test was to attempt to answer the questions posed in the Introduction. In the following, we are going to show how the techniques described can help in finding the answers to those questions. We analyzed several data sets but only the typical behavior will be presented, leaving broader coverage of the data and related conclusions to a separate publication.
As already indicated above, even though the fluctuations in the heart rate variability are clearly visible in the effective Holder exponent data, they will benefit from filtering with the moving average filter. The moving average, Eq. (5.1) is performed directly on the subsequent ordered maxima, each carrying the corresponding effective Holder exponent value. The number of maxima at the lowest scale of analysis is only roughly related to the number of heartbeats in the time series. However, this problem is negligible for the large length of averaged exponent values (wo use 100, 1000, $10000 \mathrm{ex}$ ponent values, i.e. maxima lines). Since the actual temporal information can be associated with each maximum value, we map it back on the average values obtained from the moving average and use it for the abscissa of the plots. Additionally, for each moving average window, we calculate the standard deviation from the rrean value. This standard deviation, $\mathrm{Eq}$. (5.2) is closely related to the width of histograms of the local effective Holder exponent. Therefore it also reflects the width of the multifractal spoctra over the MA-base - the range of the moving average window.

\subsection{Ad. 1)}

We have included in the same plot, Fig. 7, the result of running exactly the same procedure on a random noise sample and on the heartbeat rate. The resulting fluctuations for the random noise are much smaller in magnitude, thus supporting the observation that the fluctuations in variability of heartbeat may be of physiological or other origin and are not pure statistical fluctuations of noise data. Additionally, we checked the standard deviation of the variability, which clearly indicated a broader spectrum for the heartbeat than for the same length of white noise data.

The reader may, of course, ask the question whether the ronstationarities in the Holder exponent are the only source of wide multifractal spectrum of the heartbeat. Firstly, the nonstationarities observed show up at all temporal resolutions just as is the case for model multifracticls. It is not possible to select one single ternporal scale capturing the nonstationary behavior. Therefore, the contributions to the multifractal spectrum come from various temporal scales in comparable degree. If this were not the case, this would mean that for long base MA averages, we would get considerably 


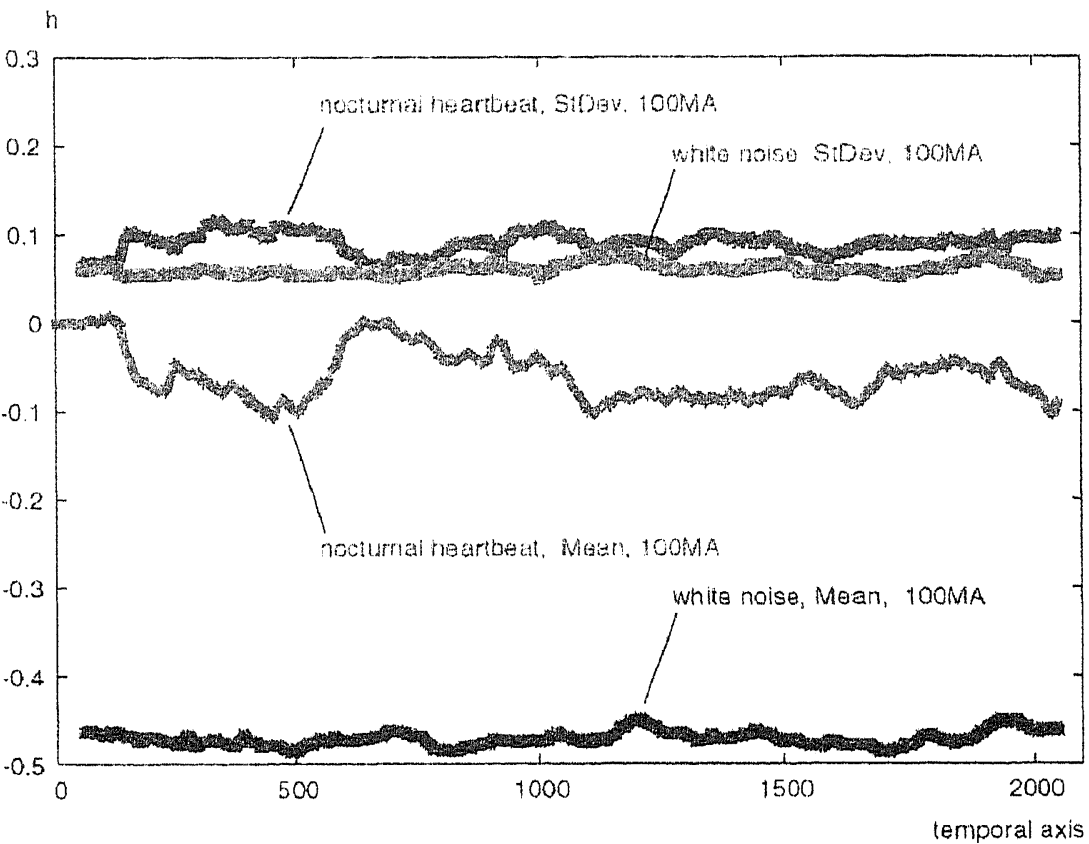

Fig. 7 Plot of the 100 base MA-smoothed effective Hölder exponent, for the heartbeat test, case and for white noise. Mean and standard deviation are shown.

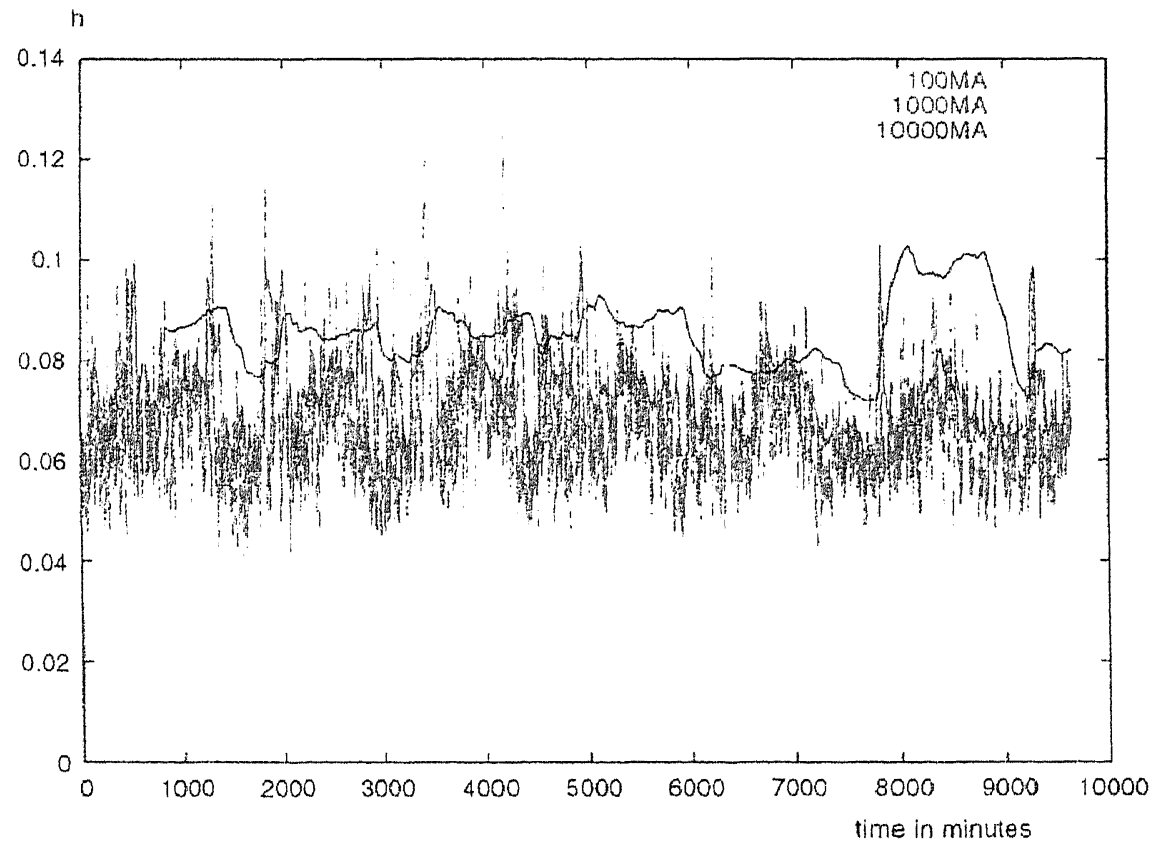

Fig. 8 Three widths of MA window, 100, 1000 and 10000 are used to test whether the fluctuations observed can be used to explain the wider multifractal spectra for the longer time series nomally used for spectra estimation (> 5000 maxima). 
higher standard deviation of the Hölder exponent than for shorter base MA averages.

Therefore, we performed a test to indicate whether the fluctuation effects observed can be used to explain the wider multifractal spectra. We used three widths of MA window - 100, 1000 and 10000 , see Fig. 8. The result of running $\mathrm{MA}$ with the longest window (approximately 2 hours record) gave a clear indication that long lengths of data result, in a wider spectra than shorter records. However, in defense of the multifractality of the heartbeat, we find in shorter base MA levels of standard devialion which are larger than those in the longer base MA. In other words, locally standard cleviation (and therefore the "local" multifractal spectrum) in $\mathrm{MA}_{100}$ exceeds that of $\mathrm{MA}_{1000}$. Similarly, locally standard deviation within a $\mathrm{MA}_{1000}$ exceeds that of MA10000. Note that it is not the plot in Fig. 8 that is being averaged. Rather, it givos the standard deviation of the result of averaging of the local effective Hölder exponent (not shown).

\subsection{Ad. 2)}

The variability plots shown in Figs. 9 and 10 come from a long rum of experiments where the test persons were given placebo or $\beta$-blocker. We analyzed two records to be referred to as "tomo" and "kou". The effective Hölder exponent was first calculated for each record and served as input for two runs of MA filter performed with 100 and 1000 maxima long window. The observed effect of the $\beta$-blocker is nil or negligible, indicating there is little change to the dynamics of the heart due to the $\beta$-blocker only (at, least for the two cases analyzed and for the $s \eta_{0}=5$ resolution considered). However, we found an interesting pattern of response to activity in these data sets. The first set shows a particularly strong response of the person in question to food. Let us remind the reader that higher values of the exponent are normally associated with a pathologic condition. The observed shift towards higher values as the result of eating (it is almost possible to estimate the volume of the meal!) may indicate some

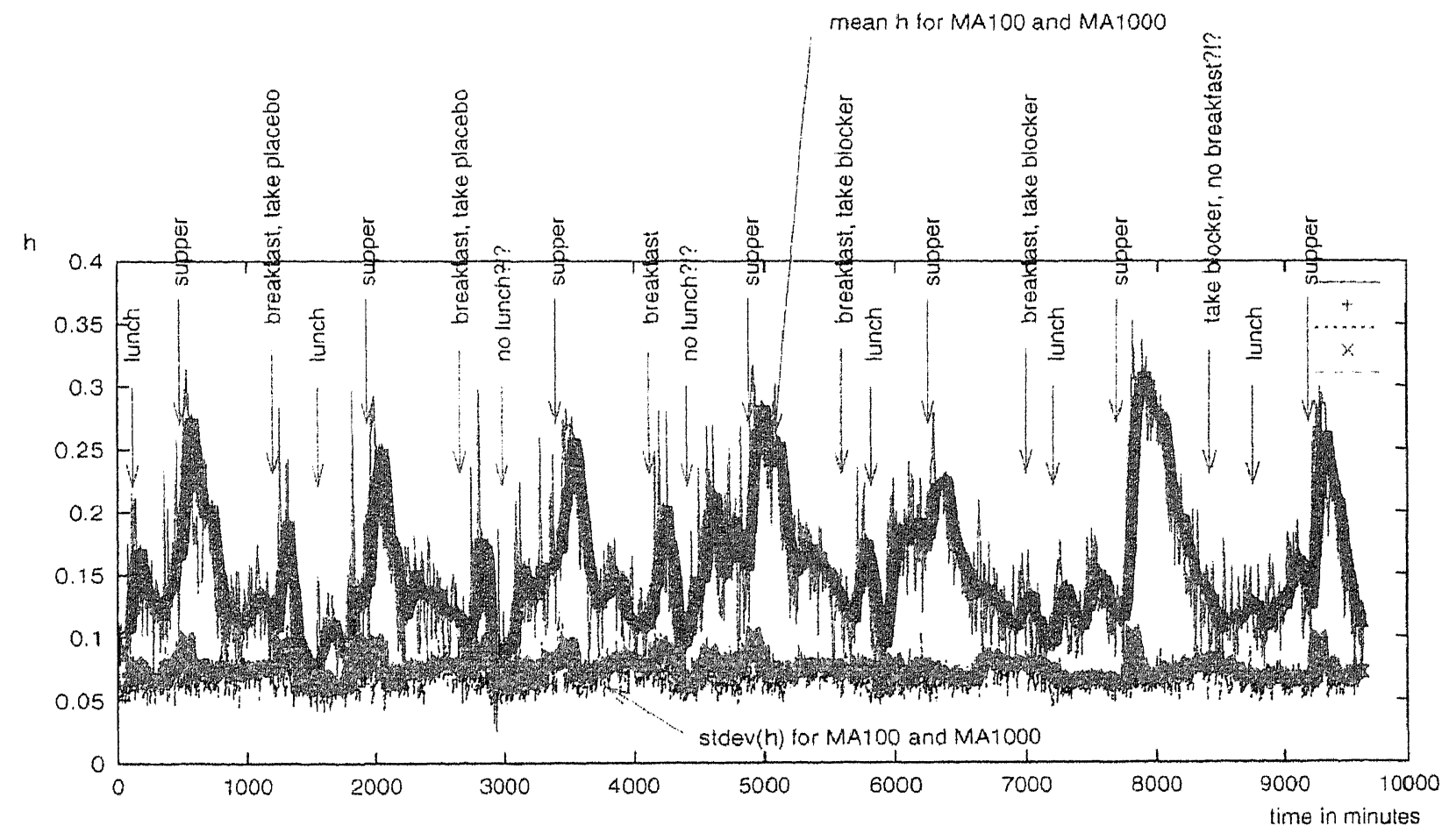

Fig. 9 The variability plot from a long run of experiments where the test persons were given placebo or $\beta$-blocker (tomo record). Two runs of MA filter were performed with 100 and 1000 maxima long window. The observed effect of the $\beta$-blocker is nil or negligible. However, an interesting pattern of response to food is evident. 


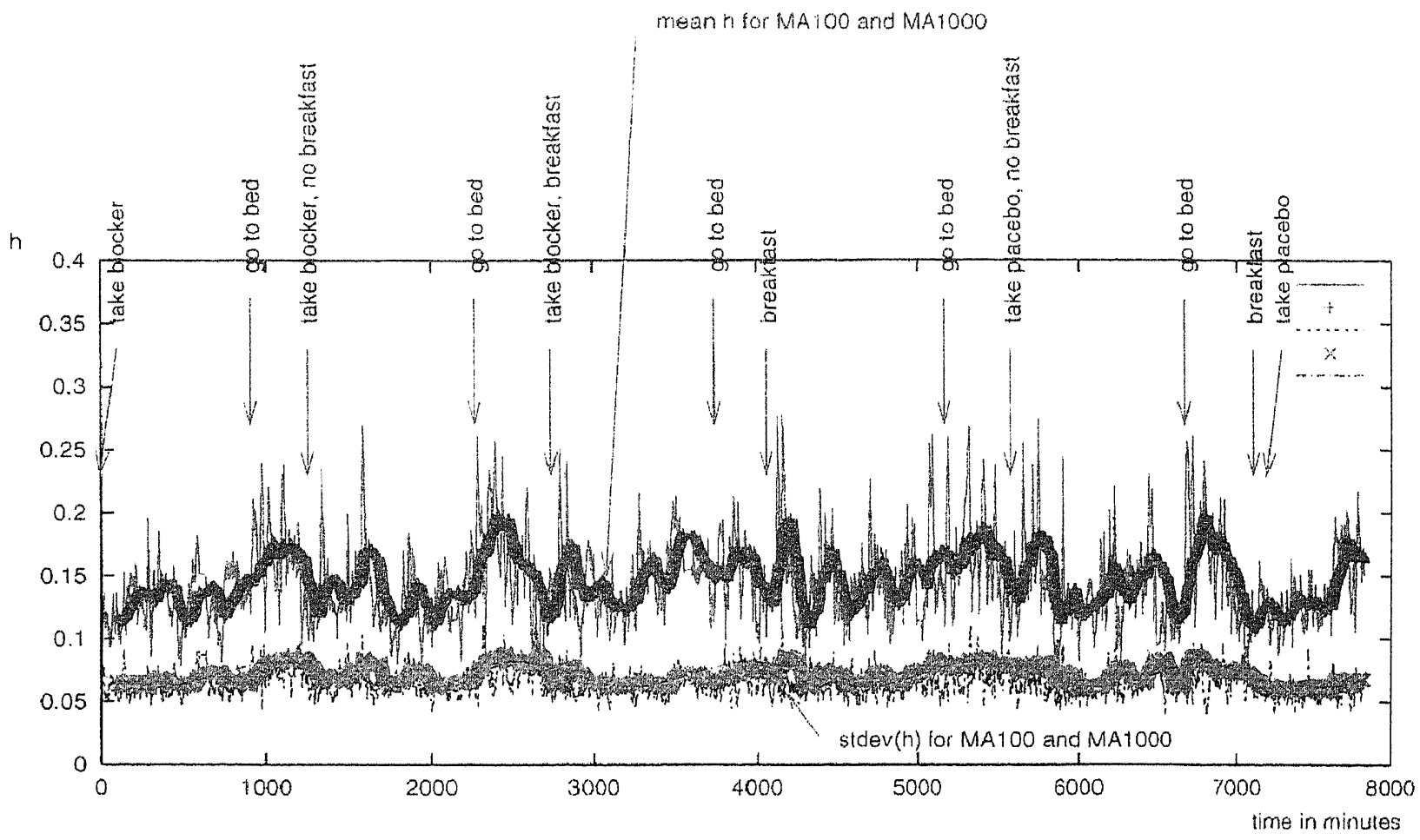

Fig. 10 Another variability plot from with the placobo or $\beta$-blocker (kou record). A.gain no response was registered. For this person, however, an interesting pattern of response to sleep has been found.

nearly pathologic response in this individual case. Another data set shows a much smaller response to food but it does show higher correlation levels during the first hours of sleep. Again this makes us speculate that the particular person may have nearly pathologic bohavior during first stages of sleep. (It should be noted that the person in study was going to bed very late 0-2 am and waking up after a relatively short sleep, 5-6 hours.)

\subsection{Ad. 3)}

Particularly in the second data set, the day/night oscillations $^{25}$ can be clearly observed in the width of the multifractal spectrum and the range of the variability (as is reflected by the standard cleviation plotted in both Figs. 9 and 10). The oscillations nearly follow the sinusoidal line (with a 24-hour period), which we superimposed on the standard deviation of the local effective Hölder exponent. The actual phase of the oscillations is shifted by some $4-5$ hours with respect to the clock. (It actually is
5-6 in the plot but about 1 hour delay connes from the MA 1000 beat base average.) This means that the ptrysiological "middle of the night" is not at 12 midnight but at $4-6$ am, similarly "middle of the day" falls at 4-6 pm, not at, 12 noon.)

In the upper plot in Fig. 11, we show the standard deviation of the local effective Hölder exponent for the tomo record. It is the enlarged plot from Fig. 9. The influence of the $\beta$-blocker on the variability range (the width of the multifractal spectrum) can be observed. Especially during the day, standard deviation of the effective Holder exponent is high, which would correspond with a wide rnultifractal spectrum calculated traditionally. This offect diminished after the placebo tablet was replaced with the $\beta$-blocker. For the last three days of the test, the standard deviation of the Holder exponent looks much smoother and seems to follow the day/night sinusoidal pattern better.

This effect is not visible in the lower plot in Fig. 11, corresponding with the kou record (enlarged from Fig. 10). In this case the $\beta$-blocker 

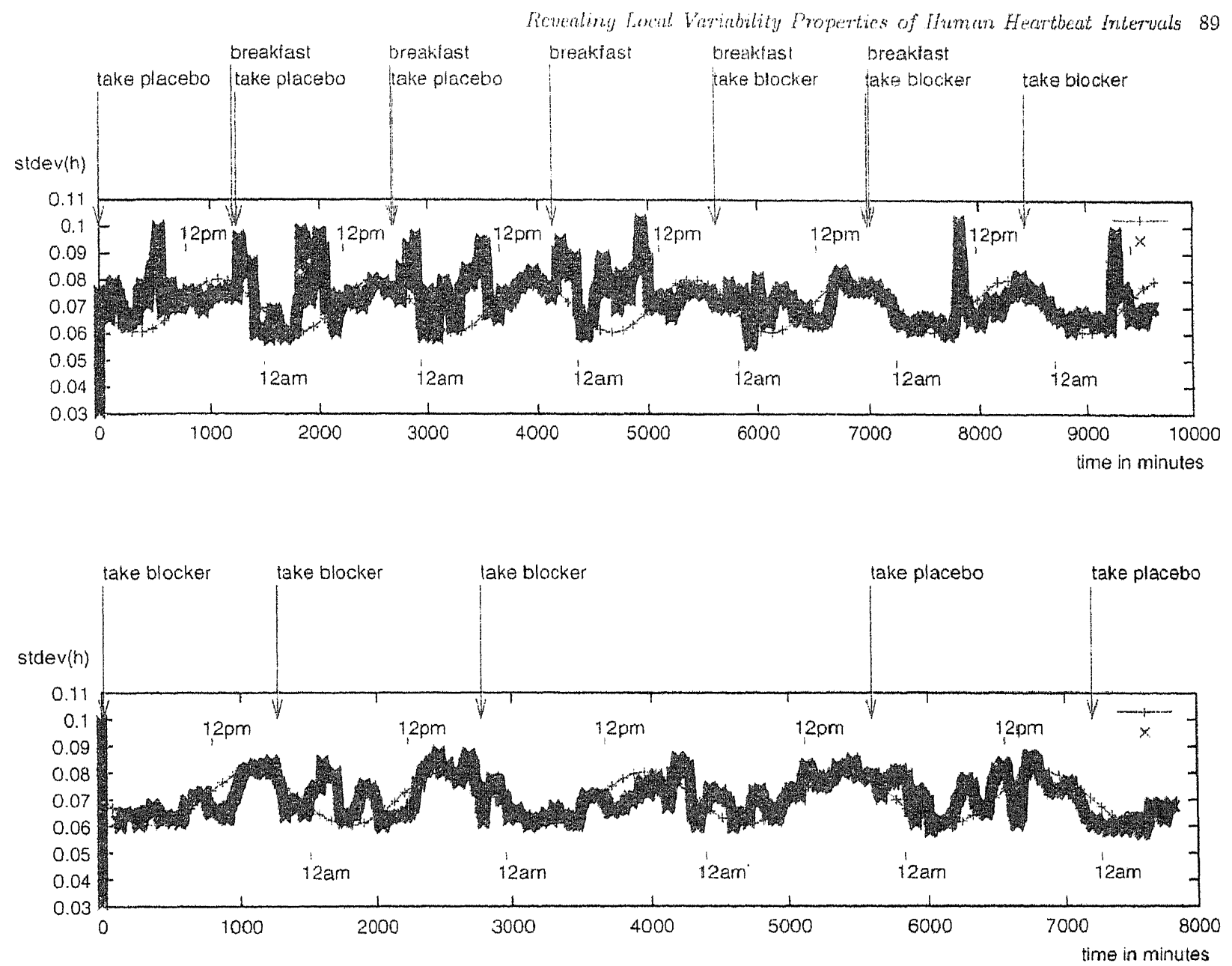

Fig. 11 Enlarged plots of standard deviation from Fig. (9) (tomo) and Fig. (10) (kou), above and below respectively. The day/night oscillations can be observed in the range of the variability as reflected by the standard deviation of the local effective Hölder exponent. The actual phase of the oscillations is shifted by sorne 4-6 hours with respect to the clock. In the upper plot, the influence of the $\beta$-blocker on the variability range (the width of the multifractal spectrum) can be observed. This is not visible in the lower plot. $(12 \mathrm{pm}=12$ miduight; $12 \mathrm{am}=12$ noon $)$.

was taken during the first few days of the test and was later replaced with placebo.

\subsection{Ad. 4)}

In Figs. 12 and 13, two samples of the sleep period taken from the tomo and kou data are displayed. The actual time period between going to bed and waking up is indicated with a line segment. A part of the sinusoidal day/night rhythm is also visible, reaching maximum somewhere near the early morning hours $(4-6 \mathrm{am})$. The mear effective Holder exponent obtained with 100 base MA is shown together with the standard deviation from the mean.
Both show large nonstationarities, different for each sample (and each night period within samples), but still considerably larger than statistical fluctuations would be in a monofractal (e.g. white) noise sample of comparable length.

The origin of these nonstationarities is not known at the moment of writing. It is possible that they are related to sleep phases, ${ }^{26}$ but this relation does not appear to be trivial and a follow-up study is expected to shed more light on this.

\subsection{Let Us Summarize}

We were able to test answers to the questions posed 
in the Introduction:

1. The observed multifractal behwior of the heartbeat is the result of "nonstationarity" of the local effective Holder exponent at all resolutions, just. as is the case with multifractals. We still do not. know the actual mechanism of these nonstationaritics. Probably different mochanisms control them at various temporal resolutions.

2. Yos, for particular temporal resolutions (wo tested about 100-1000 maxima), it is evident, that the nonstationarity can be linked with activity. The exact mechanism of this dependence musb be further studied since it cannot be validated from a limited tost of the kind presented here.

3. It seems that the influence of $\beta$-blocker is rather small and linited mainly to the standard deviation of the Hölder exponent (corresponding with the width of the multifractal spectra). In the only case where we observed such a dependence, it was narrowing the spectra width (loss "dynamic" behavior) due to the $\beta$-blocker. The $\beta$-blocker does not seem to influence the mean value of the Hölder exponent (which reflects the correlation properties of the hoartbeat).
4. We observe a full range of nonstationary behavior in the case of non-activity such as during sleep; it sems to be mainly nonstationarity of shorter time scales.

Finally, we note that the local information revealed with the effective Holder exponent seems to have potential diagnostic meaning. In particular, this holds for fluctuations revealed with the MA procelure. Their link with activity may be interesting to explore further in a diagnostic context. Of course, the global properties like the log-histogram, which can be calculated from the effective Hölder exponent, inherit the diagnostic capabilities of the $M F$ spectra calculated traditionally. ${ }^{3}$ In addition to this, it secms possible to display the mapping

$$
h_{\mathrm{MA}_{n}}(i) \rightarrow S D h_{\mathrm{MA}_{n}}(i)
$$

between the mean and the standard deviation for the investigated signals. The nonstationarities and their interrelation will be captured in such a map. Below we plotted it for two test signals, tomo and kou, for two resolutions $\mathrm{MA}_{100}$ and $\mathrm{MA}_{1000}$ [Figs. 14(a) and (b), respectively]. The ranges,

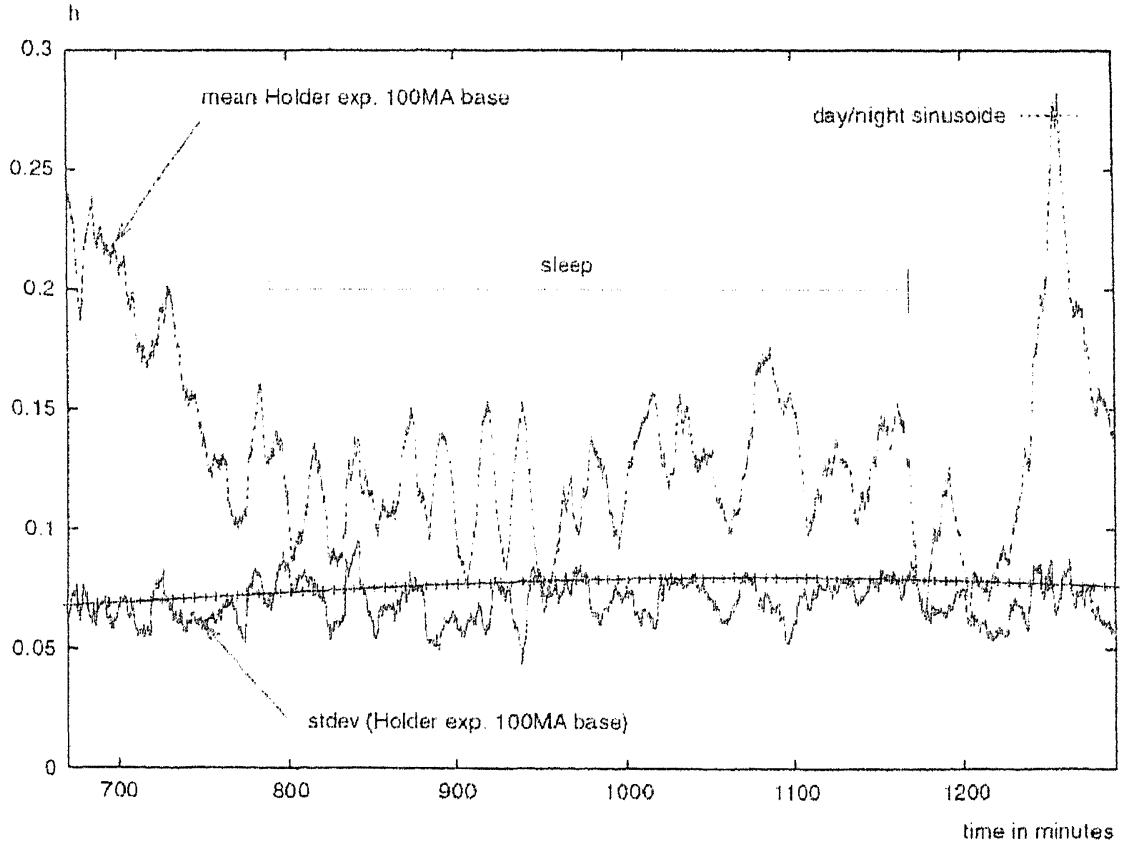

Fig. 12 A sample of the sleep period taken from the tomo data. The time period between going to bed and waking up is indicated with a line segment. The mean effective Holder exponent obtained with 100 base MA is shown touether with the standard deviation from the mean. Large nonstationarities are visible. A part of the sinusoidal day/right rhythrn is also visible. 
h

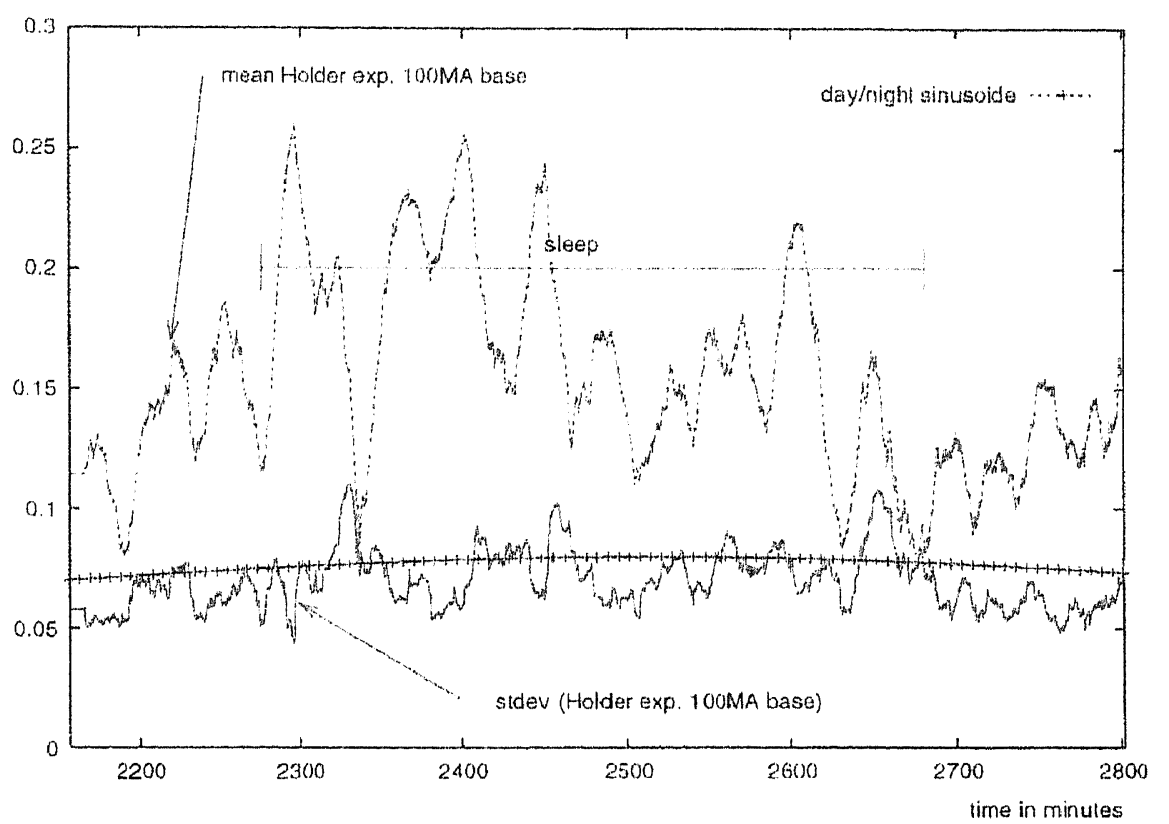

Fig. 13 A sample of the sleep period taken from the kou data. The tine period between going to bed and waking up is indicated with a line segment. The mean effective Hölder exponent obtained with 100 base MA is shown together with the standard deviation from the mean. Large nonstationarities are visible. A part of the sinusoidal day/night rhythro is also visible.

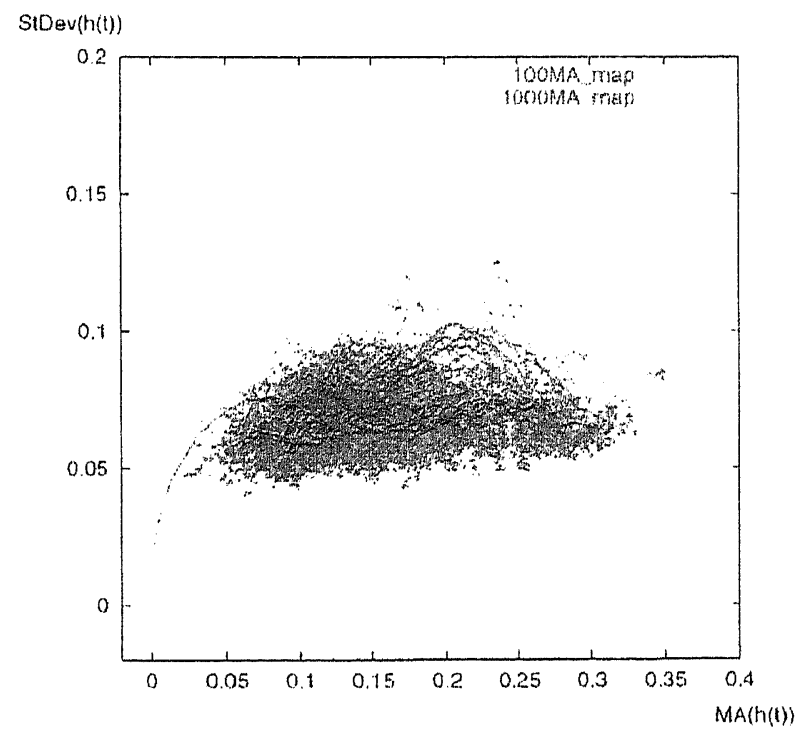

(a)

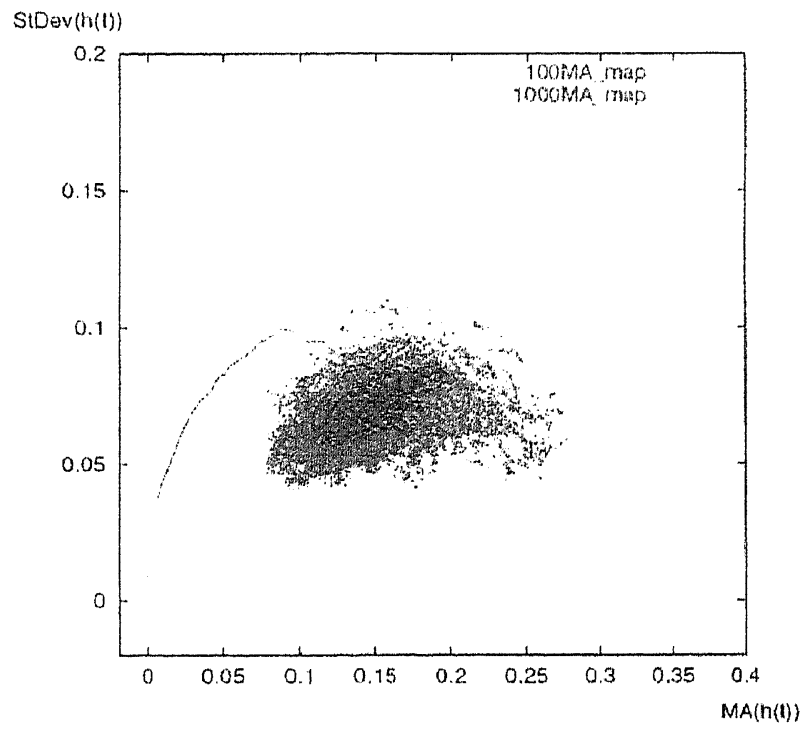

(b)

Fig. 14 Standard deviation versus mean maps for tomo (a) and kou (b). 100 and 1000 MA bases are plotted with different colors. The ranges, shape and compactness (or the degree of scatter) of these plots clearly differ for both records and may have diagnostic meaning. (Line going to 0.0 is due to finite sample size.) Note that both characteristics are not of the input signal but its local effective Hölder exponent, and therefore, can be compared without normalizing. Due to the very fire dot size, the local dot density in these maps can be readily porceived. In general, binning the dots may be required, especially for longer time series records. 
shape and compantess (or the dewed of scatter) of these plots clonly difter for both recurds, which suggests that they may have diagnostic moaning (Fig. 14).

\section{CONCLUSIONS}

The local effective Hölder exponent has been applied to evaluate variability of heart rate locally at an arbitrary position (time) and resolution (scale). The variability so obtained is compatible in the sense of clistribution to the multifractal spectra of the analyzed heart rate time series. This provides the possibility to standardize the variability estimation for comparison between different patients and between different recordings for one patient.

In addition to this, we observed intriguing patterns on nonstationary behavior of the local effective Hölder exponent. These can be related to individual response in variability records to claily activities.

We have attempted to build a methodological approach aiming at revealing such nonstationarities in local variablity at various time scales. A moving average filtering of Hölder exponent-based variability estimates was used to enhance these fluctuations/nonstationarities.

We find that this way of local presentation of scaling properties may be of clinical importance. But ultimately, we believe that the findings and methodology presented open a way better to address the question of physiological reasons for apparent multifractal behavior of the heartbeat.

\section{ACKNOWLEDGMENTS}

The author would like to thank Plamen Ivanov for discussions, especially in the context of MA filtering of the Hölder exponent. Help with the heartbeat data ${ }^{17}$ is also greatly appreciated. Thanks to Gene Stanley for encouraging the color panels idea, in particular in the blind tests. Special thanks go to Yoshi Yamamoto for kindly providing long records of heartbeat. Discussions with Stephane Jaffard and Maria Haase on the negative Hölder exponent are greatly appreciated. Many thanks to Tamas Vicsek, whose comments led to considerable improvement in the final version of the paper. Arno Siebes, thanks for supporting this work. This work has been carried out with the financial support from the Impact project.

\section{REWERENCES}

1. Z. R. Struzik, "Local Effective Hölder Exponent Estimation on the Wavelet Transform Maxima Tree," in Fractals: Theory and Applications in Engineering, eds. M. Dekking, J. Lévy Véhel, E. Lutton and C. Pricot (Springer-Verlag, 1999), pp. 93-112

2. Z. R. Struzik, "Determining Local Singularity Strengths and Their Spectra with the Wavelet Transform," Fractals 8(2), 163-179 (2000).

3. P. Ch. Ivanov, M. G. Rosenblum, L. A. Nunes Antual, Z. R. Struzik, S. Havlin, A. L. Goldberger and H. F. Stanley, "Multifractality in Human Heartheat Dynamics," Nature 399, 461-465 (1999).

4. M. Kobayashi and T. Musha, "I/f Fluctuation of Heartbeat Period," IEEE Trans Biomed. Eng. 29, 456-457 (1981).

5. C.-K. Peng, J. Mietus, J. M. Hausdorff, S. Hewlin, H. E. Stanley and A. L. Goldberger, "Long-Range Anticorrelations and Non-Gaussian Behavior of the Heartbeat," Phys. Reu. Lett. 70, 1343-1346 (1993).

6. J. B. Bassingthwaighte, L. S. Liebovitch and B. J. West, Fractal Pluysiology (Oxford University Press, 1994).

7. R. C. Turceot, M. C. Teich, "Fractal Character of the Electrocardiogram: Distinguishing Heart-Failure and Normal Patients," Ann. Biomed. Eng. 24, 269293 (1990).

8. M. Meyer, "Scaling Properties of Heartbeat Interval Fluctuations in Fealth and Disease," in Fractals and Beyond, ed. M. M. Novak (World Scientific, 1998), pp. $33-42$.

9. A. Arneodo, E. Bacry and J. F. Muzy, "Wavelets and Multifractal Formalism for Singular Signals: Application to Turbulence Data," PRL 67(25), 3515 (1991).

10. A. Arneodo, E. Bacry and J. F. Muzy, "The Thermodynamics of Fractals Revisited with Wavelets," Physica A.213, 232 (1995); J. F. Muzy, E. Bacry and A. Arneodo, "The Multifractal Formalism Revisited with Wavelets," Int. I. Bifurc. Chaos 4(2), 245 (1994).

11. A. Arneodo, A. Argoul, J. F. Muzy, M. Tabard and E. Bacry, "Beyond Classical Multifractial Analysis using Wavelets: Uncovering a Multiplicative Process Hidden in the Geometrical Complexity of Diffusion Limited Aggregates," Fractals 1, 629 (1995).

12. A. Arneodo, E. Bacry, P. V. Graves and J. F. Muzy, "Characterizing Long- Range Correlations in DNA Sequences from Wavelet Analysis," PRL 74(16), 3293 (1995).

13. A. Davis, A. Marshak and W. J. Wiscombe, "Wavelet-Based Multifractal Analysis of Nonstationary and/or Intermittent Geophysical Signals," in Wavelets in Geophysics, eds. E. FoufoulaGeorgiou and P. Kumar (Academic Press, 1994), pp. 249-298. 
14. M. Farge, N. K.-R.. Kevlahan, V. Perrier, "Turbulence Analysis, Modelling and Computing Using Wavelets," in Wavelets in Physics, ed. J. C. van den Berg (Cambridge Universily Press, 1999), pp. $117 \cdots 200$

15. P. Ch. Ivanov, M. G. Rosenblum, G.-K. Peng, J. Mietus, S. Havlin, H. E. Stanley and A. L. Goldberger, "Scaling Behaviour of Heartbeat Intervals Obtained by Wavelet-Based Time-Series Analysis," Nature 383, 323 (1996).

16. 1. Daubechies, Ten Lectures on Wuvelets (S.I.A.M., 1992)

17. M. Holschneider, Wavelets -... An Aralysis Tool (Oxford Science Publications, 1995).

18. S. Mallat, A Wavelet Tour of Signal Processing (Acadernic Press, 1998).

19. S. Jaffard, "Multifractal Formalism for Functions: I. Results Valid for all Functions, II. Self-Similar Functions," SIAM J. Math. Anal. 28(4), 944-998 (1997).

20. S. Mallat and W. L. Hwang, "Singularity Detection and Processing with Wavelets," IEEE Truns. In.f. Th. 38, 617 (1992).
21. S. Mallat and S. Zhong, "Complete Signal Representation with Multiscale Edges," IEEE Trans. PAMI $14,710(1992)$.

22. Z. R. Struaik, "The Wavelet Transform in the Solution to the Invorse Fractal Problem," Fractals 3(2), 329 (1995); Z. R. Struzik, From Coastline Length to Inverse Fractal Problem: The Concept of Fractal Metroloyy, Thesis, University of Amsterdam (1996).

23. A. Arneodo, E. Bacry and J. F. Muzy, "Solving the Inverse Fractal Problem from Wavelet Analysis," Europhys. Lett. 25(7), 479-484 (1994).

24. hittp://www. physionet.org/physiobank

25. P. Ch. Ivanov, A. Bunde, L. A. N. Amaral, J. FritschYello, R. M. Baevsky, S. Havlin, H. E. Stanley and A. L. Goldberger, "Sleep-Wake Differences in Scaling Behavior of the Human Heartbeat: Analysis of Terrestrial and Long-Term Space Flight Data," Europhys. Lett. 48, 594-600 (1999).

26. T. Penzel, A. Bunde, J. Heitmann, J. W. Kantelhardt, J. H. Peter \& K. Voigt, "Sleep-StageDependent Heart Rate Variability in Patients with Obstructive Slcep Apnea," in Computers in Cardiology $26,249-252(1999)$. 